\title{
A new Proof of Desingularization over fields of characteristic zero
}

\author{
Santiago Encinas and Orlando Villamayor
}

\begin{abstract}
We present a proof of embedded desingularization for closed subschemes which does not make use of Hilbert-Samuel function and avoids Hironaka's notion of normal flatness (see also [17] page 224). Given a subscheme defined by equations, we prove that embedded desingularization can be achieved by a sequence of monoidal transformations; where the law of transformation on the equations defining the subscheme is simpler then that used in Hironaka's procedure.

This is done by showing that desingularization of a closed subscheme $X$, in a smooth sheme $\mathrm{W}$, is achieved by taking an algorithmic principalization for the ideal $I(X)$, associated to the embedded scheme $X$. This provides a conceptual simplification of the original proof of Hironaka. This algorithm of principalization (of Logresolution of ideals), and this new procedure of embedded desingularization discussed here, have been implemented in MAPLE.
\end{abstract}

\section{Introduction}

In his monumental work ([20]), Hironaka proved desingularization, and a strong form of principalization, also called Log-resolution, of ideals in regular schemes; both results proved over fields of characteristic zero. His proof is existential in the sense that it does not provide an algorithm in order to achieve desingularization.

Different constructive proofs, following Hironaka's approach, appear in [7], [16], and in [27] (see also [28]). Each one of these proofs provides an algorithm of desingularization which indicates where to blow-up in order to eliminate the singularities in a step by step procedure. The idea is to define

2000 Mathematics Subject Classification: 14E15, 32S45.

Keywords: Resolution of singularities, desingularization. 
invariants of singular points, and to show that these invariants improve when blowing up the set of worst singularities. Desingularization is then achieved by repeatedly blowing up the set of worst points.

All algorithmic procedures mentioned above make use, as Hironaka did in his original work, of the Hilbert Samuel function. Namely the invariant attached to a singular point, consists of the full Hilbert Samuel function at the given point, followed by other data.

The purpose of this work is to show that embedded desingularization can be achieved in a different way, where the Hilbert Samuel functions are avoided. In fact we show that desingularization follows from algorithmic principalization.

Here principalization of ideals is meant in a strong form, in which the ideal becomes locally monomial after a suitable sequence of monoidal transformations (also called Log-resolution of ideals). Algorithmic principalization of ideals, is stated here in 4.4 as an algorithm of resolution of basic objects (see also 2.5).

This paper is organized so that the reader can easily get into what is new in this proof, without having to go through technicalities. To this end we do not include here details of algorithmic resolution of basic objects; here we focus on showing why our short proof in 4.8 arises quite directly from properties extracted from the algorithm. Let us mention that this algorithm of resolution of basic objects treated in [17], and implemented in MAPLE by Bodnár and Schicho, is available at

http://www.risc.uni-linz.ac.at/projects/basic/adjoints/blowup and we encourage the reader to test on examples.

This proof has also led to stronger formulations of desingularization in [12], and we also mention [14] for a proof in a different line. Applications of our proof to the study of desingularization of families of embedded schemes were developed in [15].

In this paper we address equivariant desingularization for equidimensional schemes embedded in smooth schemes. Those willing to postpone our discussion on equivariance should skip Definition 1.4 and all Section 3.

The contents of this paper are also included in Matsuki's clear and detailed discussion of algorithmic desingularization (see [24]).

We finally refer to [11] for an extension of this proof to the class of locally embedded excellent schemes, and for a full description of this equivariant algorithm in that more ample context. That paper also includes the stronger form of desingularization introduced in [13], the result of desingularization of families of embedded schemes in [15], and other applications of the theorem. 


\section{Embedded desingularization}

Definition 1.1. Let $W$ be a regular scheme and let $Y_{1}, \ldots, Y_{k} \subset W$ be a set of closed subschemes. We say that $Y_{1} \cup \ldots \cup Y_{k}$ have normal crossing at a point $\xi \in W$ if there exists a regular system of parameters $\left\{x_{1}, \ldots, x_{d}\right\} \subset \mathcal{O}_{W, \xi}$, such that for each $i \in\{1, \ldots, k\}$, either $\mathcal{I}\left(Y_{i}\right)_{\xi}=\mathcal{O}_{W, \xi}$, or

$$
\mathcal{I}\left(Y_{i}\right)_{\xi}=\left\langle x_{i_{1}}, \ldots, x_{i_{s_{i}}}\right\rangle
$$

for some $x_{i_{1}}, \ldots, x_{i_{s_{i}}} \in\left\{x_{1}, \ldots, x_{d}\right\}$. We say that $Y_{1} \cup \ldots \cup Y_{k}$ have normal crossing in $W$ if they have normal crossing at any point of $W$.

Definition 1.2. Let $W$ be a pure dimensional scheme, smooth over a field $\mathbf{k}$ of characteristic zero, and let $E=\left\{H_{1}, \ldots, H_{r}\right\}$ be a set of smooth hypersurfaces in $W$ with normal crossing. The couple $(W, E)$ is said to be a pair.

1.3. Transformation of pairs. A regular closed subscheme $Y \subset W$ is said to be permissible for a pair $(W, E)$ if $Y$ has normal crossing with $E$ (i.e. with $\left.\cup_{i=1}^{r} H_{i}\right)$.

Given $(W, E)$ and $Y$ as above, let

$$
W \longleftarrow W_{1}
$$

be the blow up at $Y$, and set $E_{1}=\left\{H_{1}^{\prime}, \ldots, H_{r}^{\prime}, H_{r+1}^{\prime}\right\}$, where $H_{i}^{\prime}$ denotes the strict transform of $H_{i}$, and $H_{r+1}^{\prime}=\Pi^{-1}(Y)$ is the exceptional hypersurface in $W_{1}$. The permissibility of $Y$ insures that $W_{1}$ is smooth, and that $E_{1}$ has normal crossing. We say that

$$
(W, E) \longleftarrow\left(W_{1}, E_{1}\right)
$$

is a transformation of pairs defined by the permissible center $Y$.

A sequence of transformations of pairs:

$$
\left(W_{0}, E_{0}\right) \longleftarrow\left(W_{1}, E_{1}\right) \longleftarrow \cdots \longleftarrow\left(W_{k}, E_{k}\right)
$$

with centers $Y_{i}, i=0,1, \ldots, k-1$ is a composition of transformations.

Definition 1.4. We say that an isomorphism $\Theta: W_{0} \rightarrow W_{0}$ defines an isomorphism on the pair $\left(W_{0}, E_{0}\right)$ if $\Theta\left(H_{i}\right) \subset H_{i}$ for any $H_{i} \in E_{0}$.

A group $G$ is said to act on a pair $\left(W_{0}, E_{0}\right)$, if it acts on $W_{0}$, and if any $\Theta \in G$ defines an isomorphism on the pair.

Let now

$$
\left(W_{0}, E_{0}\right) \longleftarrow\left(W_{1}, E_{1}\right)
$$

be a transformation of pairs defined by a permissible center $Y \subset W_{0}$. Assume that a group $G$ acts both on the pair $\left(W_{0}, E_{0}\right)$ and also on $Y \subset W_{0}$. In this case $G$ also acts on the pair $\left(W_{1}, E_{1}\right)$ ([11, Lemma 4.2]). 
A sequence of transformations of pairs (1.3.1) is said to be $G$-equivariant if $G$ acts on the pairs $\left(W_{i}, E_{i}\right), 0 \leq i \leq k-1$, and $\Theta\left(Y_{i}\right)=Y_{i}$ for $0 \leq i \leq k-1$, for any $\Theta \in G$. In such case the group $G$ acts on the pair $\left(W_{k}, E_{k}\right)$.

Note that by a step by step lifting of the action, we can also state that (1.3.1) is equivariant if $G$ acts on acts on the pair $\left(W_{0}, E_{0}\right)$, and also on the centers $Y_{i}$ for $0 \leq i \leq k-1$.

Theorem 1.5 (Embedded Desingularization). Given a closed reduced and equidimensional subscheme $X_{0} \subset W_{0}$, there is a sequence of transformations of pairs

$$
\left(W_{0}, E_{0}=\emptyset\right) \longleftarrow \cdots \longleftarrow\left(W_{r}, E_{r}\right)
$$

inducing a proper birational morphism $\Pi: W_{r} \longrightarrow W_{0}$, so that setting $\operatorname{Sing}\left(X_{0}\right)$ as the singular locus of $X_{0}, \operatorname{Reg}\left(X_{0}\right)=X_{0}-\operatorname{Sing}\left(X_{0}\right)$, and $X_{r} \subset W_{r}$ the strict transform of $X_{0}$, then:

(i) The morphism $\Pi$ defines an isomorphism

$$
W_{0} \backslash \operatorname{Sing}\left(X_{0}\right) \cong W_{r} \backslash \bigcup_{H \in E_{r}} H
$$

and hence $\operatorname{Reg}(X) \cong \Pi^{-1}(\operatorname{Reg}(X)) \subset X_{r}$ via $\Pi$.

(ii) $X_{r}$ is regular and has normal crossing with $E_{r}$.

(iii) (Equivariance) Any action of a group $G$ on $X_{0} \subset W_{0}$ has a unique natural lifting to an action on $\left(W_{r}, E_{r}\right)$ and on $X_{r} \subset W_{r}$ (3.1).

Proof: See 4.8 .

\section{Basic Objects}

2.1. We will prove Theorem 1.5 as a corollary of the algorithm of principalization. We will essentially unify principalization and desingularization by means of the notions of basic objects and of algorithmic resolution of basic objects, which we now introduce.

Definition 2.2. [16, Definition 1.2] A basic object $\left(W_{0},\left(J_{0}, b\right), E_{0}\right)$, is a pair $\left(W_{0}, E_{0}\right)$, an ideal $J_{0} \subset \mathcal{O}_{W_{0}}$, and a positive integer $b$. We require that $\left(J_{0}\right)_{\xi} \neq 0$ for any $\xi \in W_{0}$.

Note here that $\mathcal{O}_{W_{0}, \xi}$ is a local regular ring; let $\boldsymbol{\nu}_{J_{0}}(\xi)$ denote the order of $\left(J_{0}\right)_{\xi}$ at the local ring $\mathcal{O}_{W_{0}, \xi}$ (the biggest integer such that the corresponding power of the maximal ideal contains $\left.\left(J_{0}\right)_{\xi}\right)$.

We finally define

$$
\operatorname{Sing}\left(J_{0}, b\right)=\left\{\xi \in W_{0} \mid \boldsymbol{\nu}_{J_{0}}(\xi) \geq b\right\} \quad\left(\subset W_{0}\right),
$$

which is a closed subset in $W_{0}$. 
2.3. Transformation of basic objects. [16, Definition 1.4] We shall say that $Y_{0}$ is permissible for the basic object $\left(W_{0},\left(J_{0}, b\right), E_{0}\right)$ if $Y_{0}$ is permissible for the pair $\left(W_{0}, E_{0}\right)$, and, in addition, $Y_{0} \subset \operatorname{Sing}\left(J_{0}, b\right)$. In such case let $W_{0} \longleftarrow W_{1}$ be the blow-up with center $Y_{0}$ and denote by $H_{1} \subset W_{1}$ the exceptional hypersurface. In the particular case in which $Y_{0}$ is irreducible, with generic point $\xi$, then $\xi \in \operatorname{Sing}\left(J_{0}, b\right)$ and $\boldsymbol{\nu}_{J_{0}}(\xi) \geq b$ (since $Y_{0} \subset \operatorname{Sing}\left(J_{0}, b\right)$ ).

Set $c_{1}=\boldsymbol{\nu}_{J_{0}}(\xi)$; there is a factorization

$$
J_{0} \mathcal{O}_{W_{1}}=I\left(H_{1}\right)^{c_{1}} \bar{J}_{1}
$$

for a well defined sheaf of ideals $\bar{J}_{1} \subset \mathcal{O}_{W_{1}}$. In the general case, in which $Y_{0}$ is not necessarily irreducible, we obtain, in a similar way, a well defined expression as above, where now $c_{1} \geq b$ is locally constant on $H_{1}$.

We define

$$
J_{1}=I\left(H_{1}\right)^{c_{1}-b} \bar{J}_{1}
$$

and set

$$
\left(W_{0},\left(J_{0}, b\right), E_{0}\right) \longleftarrow\left(W_{1},\left(J_{1}, b\right), E_{1}\right)
$$

which we call a transformation of basic objects.

It should be noted, that in general, the sheaf of ideals $J_{1}$ is not the strict transform of $J_{0}$.

Definition 2.4. A sequence of transformations of basic objects

$$
\left(W_{0},\left(J_{0}, b\right), E_{0}\right) \longleftarrow \cdots \longleftarrow\left(W_{k},\left(J_{k}, b\right), E_{k}\right)
$$

is a resolution of $\left(W_{0},\left(J_{0}, b\right), E_{0}\right)$ if $\operatorname{Sing}\left(J_{k}, b\right)=\emptyset$.

2.5. Assume, for simplicity, that $E_{0}=\emptyset$ so that $\cup H_{i}\left(H_{i} \in E_{k}\right.$ in 2.4.1) is the exceptional locus of the composition $W_{0} \longleftarrow W_{k}$. It follows from the notion of transformation of basic objects, that if (2.4.1) is a resolution then:

$$
J_{0} \mathcal{O}_{W_{k}}=J_{k} \cdot \mathcal{M}
$$

where $\mathcal{M}$ is an invertible sheaf of ideals supported on $\cup H_{i}$ (locally spanned by a monomial), and $J_{k}$ is a sheaf of ideals with order at most $b-1$ at points of $W_{k}$.

In particular, if $b=1$ in the resolution $2.4 .1, J_{k}=\mathcal{O}_{W_{k}}$ so

$$
J_{0} \mathcal{O}_{W_{k}}=\mathcal{M}
$$

Hence, the total transform of $J_{0}$ is an invertible sheaf of ideals, locally defined by a monomial (monomialization). 


\section{Equivariance}

3.1. Let $G$ be a group acting on $W$, and $X \subset W$ a subscheme; we say that $G$ acts on $X \subset W$ when the action on $W$ induces, by restriction, an action on $X$. If $X \subset W$ is simply a closed set we view it as a subscheme with the unique reduced structure.

Since we will prove both desingularization and monomialization by means of resolutions of basic objects, we discuss now the notion of group actions within that context.

Definition 3.2. Group actions on basic objects. Consider a basic object $\left(W_{0},\left(J_{0}, b\right), E_{0}\right)$ and a group $G$ acting on the pair $\left(W_{0}, E_{0}\right)$. We will say that $G$ acts on the basic object when following conditions holds:

$0)$ The group $G$ acts on $\operatorname{Sing}\left(J_{0}, b\right) \subset W_{0}$, namely

$$
\Theta\left(\operatorname{Sing}\left(J_{0}, b\right)\right)=\operatorname{Sing}\left(J_{0}, b\right)
$$

for any $\Theta \in G$.

k) Whenever a sequence of transformations of basic objects

$$
\left(W_{0},\left(J_{0}, b\right), E_{0}\right) \longleftarrow \cdots \longleftarrow\left(W_{k},\left(J_{k}, b\right), E_{k}\right)
$$

is such that the induced sequence of pairs

$$
\left(W_{0}, E_{0}\right) \longleftarrow \cdots \longleftarrow\left(W_{k}, E_{k}\right)
$$

is $G$-equivariant (1.4), then the group $G$ acts on $\operatorname{Sing}\left(J_{k}, b\right) \subset W_{k}$, namely

$$
\Theta\left(\operatorname{Sing}\left(J_{k}, b\right)\right)=\operatorname{Sing}\left(J_{k}, b\right)
$$

for any $\Theta \in G$.

3.3. Main Example. Note that the previous definition involves all possible $G$-equivariant sequences of transformations of basic objects.

If a group $G$ acts on $W_{0}$, each $\Theta \in G$ defines an isomorphism $\Theta^{\#}$ : $\mathcal{O}_{W_{0}} \rightarrow \mathcal{O}_{W_{0}}$. Suppose now that a group $G$ acts on a pair $\left(W_{0}, E_{0}\right)$, and that $J_{0}$ is a is $G$-invariant sheaf of ideals (i.e. $\Theta^{\#}\left(J_{0}\right)=J_{0}$ for any $\Theta \in G$ ). We claim that in these conditions, for any $b$, the group $G$ acts on the basic object $\left(W_{0},\left(J_{0}, b\right), E_{0}\right)$.

So assume that $Y_{0}$ is permissible for the basic object $\left(W_{0},\left(J_{0}, b\right), E_{0}\right)$, and $G$-invariant. Note that $Y_{0} \subset \operatorname{Sing}\left(J_{0}, b\right)$ is permissible for the pair $\left(W_{0}, E_{0}\right)$. Set $W_{0} \longleftarrow W_{1}$ the blow-up with center $Y_{0}$, and denote by $H_{1} \subset W_{1}$ the exceptional hypersurface.

Since $Y_{0}$ is closed and regular, it is the disjoint union of irreducible components $Y_{0}=Z_{1} \cup \cdots \cup Z_{s}$, each $Z_{i}$ with generic point, say $\xi_{i} \in$ $\operatorname{Sing}\left(W_{0}, b\right)$. Note that $H_{1}$ is also a union of $s$ irreducible components, say $H_{1}=V_{1} \cup \cdots \cup V_{s}$. 
Let

$$
J_{0} \mathcal{O}_{W_{1}}=I\left(H_{1}\right)^{c_{1}} \bar{J}_{1} \text { and } \quad J_{1}=I\left(H_{1}\right)^{c_{1}-b} \bar{J}_{1}
$$

be as in 2.3 , and note that the locally constant function $c_{1}$ is constant and equal to $\boldsymbol{\nu}_{J_{0}}\left(\xi_{i}\right)$ along each component $V_{i}$.

On the other hand, the group $G$ acts on the set $\left\{\xi_{1}, \ldots, \xi_{s}\right\}$, and if $\Theta\left(\xi_{i}\right)=\xi_{j}$ for some $\Theta \in G$, then $\boldsymbol{\nu}_{J_{0}}\left(\xi_{i}\right)=\boldsymbol{\nu}_{J_{0}}\left(\xi_{j}\right)$, since $\Theta\left(J_{0}\right)=J_{0}$.

Since the action of $G$ can be lifted to $W_{1}$, and since $J_{0}$ is $G$-invariant, it follows that the total transform $J_{0} \mathcal{O}_{W_{1}}$ is $G$-invariant.

We leave it to the reader to check that the sheaves of ideals $I\left(H_{1}\right)^{c_{1}}$, $I\left(H_{1}\right)^{c_{1}-b}$, and $J_{1}$ are $G$-invariant in $W_{1}$. In particular $G$ acts on $\operatorname{Sing}\left(J_{1}, b\right) \subset$ $W_{1}$. A step by step argument shows now that $G$ acts on the basic object $\left(W_{0},\left(J_{0}, b\right), E_{0}\right)$ in the sense of 3.2 .

Take for instance a group $G$ acting on $X \subset W(3.1)$, where $X$ is a subscheme defined by a sheaf of ideals $J$. In this case $J$ is $G$-invariant, so $G$ acts on the basic object $(W,(J, 1), \emptyset)$.

\section{Algorithms of Desingularization}

4.1. We now discuss the notion and properties of algorithmic resolution of basic object, which will lead us to a constructive proof of the theorem of desingularization.

Definition 4.2. Fix a totally ordered set $(I, \leq)$, and a closed set $F \subset W$. A function $h: F \longrightarrow I$ is said to be upper-semi-continuous if:

i) $h$ takes only finitely many values, and

ii) $\{\xi \in F \mid h(\xi) \geq \alpha\}$ is a closed set, for any $\alpha \in I$.

We denote by $\max h$ the maximum value in $I$ achieved by $h$, and set Max $h=$ $\{\xi \in F \mid h(\xi)=\max h\}$ which is closed in $F$.

Given a basic object $(W,(J, b), E)$, we say that an upper-semi-continuous function

$$
h: \operatorname{Sing}(J, b) \longrightarrow I
$$

is equivariant if, for any group $G$ acting on this basic object,

$$
h(\xi)=h(\Theta(\xi)) \quad \forall \xi \in \operatorname{Sing}(J, b) \quad \forall \Theta \in G .
$$

4.3. Note that if $h$ is equivariant, then any group $G$ acting on the basic object $(W,(J, b), E)$ also acts on the closed set $\underline{\operatorname{Max}} h \subset W$. If, in addition, Max $h$ is permissible for $(W, E)$, then any such $G$ also acts on the transform of the basic object with center $\underline{\operatorname{Max}} h$. 
4.4. Algorithm of resolution of basic objects. Let $d$ be a non-negative integer. An algorithm of resolution for $d$-dimensional basic objects consists of:

A: A totally ordered set $\left(I_{d}, \leq\right)$.

B: For each basic object $\left(W_{0},\left(J_{0}, b\right), E_{0}\right)$ with $d=\operatorname{dim} W_{0}$ :

i: An equivariant function $f_{0}^{d}: \operatorname{Sing}\left(J_{0}, b\right) \longrightarrow I_{d}$ is defined, and this function has the property that $\underline{\operatorname{Max}} f_{0}^{d} \subset \operatorname{Sing}\left(J_{0}, b\right)$ is permissible for $\left(W_{0},\left(J_{0}, b\right), E_{0}\right)$. Suppose, by induction, that an equivariant sequence with centers $Y_{i} \subset \operatorname{Sing}\left(J_{i}, b\right), i=0, \ldots, r-1$ :

$\left(W_{0},\left(J_{0}, b\right), E_{0}\right) \leftarrow \cdots \leftarrow\left(W_{r-1},\left(J_{r-1}, b\right), E_{r-1}\right) \leftarrow\left(W_{r},\left(J_{r}, b\right), E_{r}\right)$

together with equivariant functions $f_{i}^{d}: \operatorname{Sing}\left(J_{i}, b\right) \longrightarrow I_{d}, i=$ $0, \ldots, r-1$ have been defined, and that $Y_{i}=\underline{\operatorname{Max}} f_{i}^{d}$. Then:

ii: If $\operatorname{Sing}\left(J_{r}, b\right) \neq \emptyset$, an equivariant function $f_{r}^{d}: \operatorname{Sing}\left(J_{r}, b\right) \longrightarrow I_{d}$ is defined, and $\underline{\operatorname{Max}} f_{r}^{d}$ is permissible for $\left(W_{r},\left(J_{r}, b\right), E_{r}\right)$.

Note that $\mathbf{B}(\mathbf{i i})$ says that whenever $\operatorname{Sing}\left(J_{r}, b\right) \neq \emptyset$ there is an equivariant enlargement of (4.4.1) with center $Y_{r}=\underline{\operatorname{Max}} f_{r}^{d}$ (see 4.3).

C: For some index $r$, depending on the basic object $\left(W_{0},\left(J_{0}, b\right), E_{0}\right)$, the equivariant sequence constructed in $\mathbf{B}$ is a resolution (i. e. $\left.\operatorname{Sing}\left(J_{r}, b\right)=\emptyset\right)$.

4.5. We refer the reader to [8] for an implementation of the algorithm treated in [17, Theorem 7.13], to see how it works on examples.

Condition $\mathbf{C}$ says that for $i=0,1, \ldots, k$, the functions $f_{i}: \operatorname{Sing}\left(J_{i}, b\right) \rightarrow$ $I_{d}$ define a resolution of the basic object $\left(W_{0},\left(J_{0}, b\right), E_{0}\right)$, with centers $\underline{\operatorname{Max}} f_{i}^{d}$. We will refer to it as the resolution defined by the algorithm, or the resolution defined by the functions $f_{i}$. Note that $\mathbf{B}$ says that this resolution is equivariant.

Let $\mathcal{B}=\left(W_{0},\left(J_{0}, b\right), E_{0}\right)$ be a basic object. If $U_{0} \subset W_{0}$ is a non-empty open set, then we set the restriction of the basic object to be

$$
\left(W_{0},\left(J_{0}, b\right), E_{0}\right)_{U_{0}}=\left(U_{0},\left(\left.J\right|_{U_{0}}, b\right), E_{U_{0}}\right),
$$

where $\left.J\right|_{U_{0}}$ is the restriction of the sheaf of ideal to $U_{0}$ and $E_{U_{0}}=\left\{H \cap U_{0} \mid\right.$ $H \in E\}$. If

$$
\left(W_{0},\left(J_{0}, b\right), E_{0}\right) \leftarrow \cdots \leftarrow\left(W_{N-1},\left(J_{N-1}, b\right), E_{N-1}\right) \leftarrow\left(W_{N},\left(J_{N}, b\right), E_{N}\right)
$$

is the resolution defined by the algorithm, it induces naturally a sequence

$$
\left(W_{0},\left(J_{0}, b\right), E_{0}\right)_{U_{0}} \leftarrow \cdots \leftarrow\left(W_{N-1},\left(J_{N-1}, b\right), E_{N-1}\right)_{U_{N-1}} \leftarrow\left(W_{N},\left(J_{N}, b\right), E_{N}\right)_{U_{N}}
$$

where each $U_{k}$ is an open subset in $W_{k}$ (the pull back of $U_{0}$ in $W_{k}$ ). 
Each function $f_{i}: \operatorname{Sing}\left(J_{i}, b\right) \rightarrow I_{d}$ induces by restriction a function on $U_{i} \cap \operatorname{Sing}\left(J_{i}, b\right)$. Note that if $\underline{\operatorname{Max}} f_{i}^{d} \cap U_{i}=\emptyset$, then

$$
\left(W_{i},\left(J_{i}, b\right), E_{i}\right)_{U_{i}} \longleftarrow\left(W_{i+1},\left(J_{i+1}, b\right), E_{i+1}\right)_{U_{i+1}}
$$

is the identity map, and hence can be neglected from (4.5.2).

4.6. Properties of the algorithm. Algorithmic principalization has the following properties (see [17, p. 192]):

p1: The functions defining the algorithmic resolution of the $d$-dimensional basic object $\left(W_{0},\left(J_{0}, b\right), E_{0}\right)_{U_{0}}$ are the restriction of the functions $f_{k}$ defining (4.5.1). In particular (4.5.2) is the resolution of $\left(W_{0},\left(J_{0}, b\right), E_{0}\right)_{U_{0}}$ defined by the algorithm.

p2: For the resolution defined by the algorithm, say (4.5.1):

$$
\max f_{0}^{d}>\max f_{1}^{d}>\cdots>\max f_{N-1}^{d} .
$$

p3: If $J_{0}$ is the ideal of a regular pure dimensional subvariety $X_{0}, E_{0}=\emptyset$ and $b=1$, then the function $f_{0}^{d}$ is constant.

p4: For any $i=0, \ldots, N-1$, the closed set $\underline{\operatorname{Max}} f_{i}^{d}$ is smooth and equidimensional. Furthermore, the dimension is determined by the value $\max f_{i}^{d}$.

4.7. It follows from Property $\mathbf{p 1})$, that if $\xi \in \operatorname{Sing}\left(J_{i}, b\right), i=0, \ldots, r-1$, and $\xi \notin Y_{i}$, then $f_{i}^{d}(\xi)=f_{i+1}^{d}\left(\xi^{\prime}\right)$ via the natural identification of the point $\xi$ with a point $\xi^{\prime}$ of $\operatorname{Sing}\left(J_{i+1}, b\right)$

4.8. Proof of theorem 1.5. Fix notation as in theorem 1.5, and consider the basic object

$$
\left(W_{0},\left(J_{0}, 1\right), E_{0}\right)
$$

where $W_{0}=W, J_{0}=\mathcal{I}(X)$ and $E_{0}=\emptyset$. Clearly $X=\operatorname{Sing}\left(J_{0}, 1\right)$.

Take $U=W \backslash \operatorname{Sing}(X)$. By p3) we know that the function $f_{0}: \operatorname{Sing}\left(J_{0}, 1\right)$ $\rightarrow\left(I_{d}, \leq\right)$ is constant on the restriction to $\operatorname{Sing}\left(J_{0}, 1\right) \cap U$ (on the restriction $\left.\left(W_{0},\left(J_{0}, 1\right), E_{0}\right)_{U}\right)$. Let $a(d)$ denote this constant value along the points in $U \cap X$.

By $4.4 \mathrm{C}$ ), we know that the algorithm provides a resolution of the basic object $\left(W_{0},\left(J_{0}, 1\right), E_{0}\right)$ by means of a finite sequence of blow-ups

$$
\left(W_{0},\left(J_{0}, 1\right), E_{0}\right) \leftarrow\left(W_{1},\left(J_{1}, 1\right), E_{1}\right) \leftarrow \ldots \leftarrow\left(W_{N},\left(J_{N}, 1\right), E_{N}\right),
$$

at permissible centers $Y_{i} \subset \operatorname{Sing}\left(J_{i}, b\right)$ for $i=0,1, \ldots, N-1$. Therefore $\operatorname{Sing}\left(J_{N}, b\right)=\emptyset$, and by p1) and p2), there must be an index $k \in$ $\{0,1, \ldots, N\}$ such that $\max f_{k}=a(d)$. Such index $k$ is unique by $\left.\mathbf{p} 2\right)$. 
Now $U$ can be identified with an open set, say $U$ again, of $W_{k}$ (note that the centers of the transformations in sequence (4.8.1) are defined by $\operatorname{Max} f_{i}$ and $\max f_{i}>a(d)$ for $i<k$ ). If $X_{k}$ denotes the strict transform of $X$ in $W_{k}$, then

$$
X_{k} \cap U=X \cap U=\underline{\operatorname{Max}} f_{k} \cap U .
$$

Since $X \cap U=\operatorname{Reg}(X)$ is dense in $X$, it follows that $X_{k}$ is the union of some of the components of $\underline{\operatorname{Max}} f_{k}$, and hence it is regular and has normal crossing with the exceptional components by Definition $4.4(\mathrm{~A})$ ). This proves (i) and (ii) of Theorem 1.5.

Now it only remains to show that the resolution of singularities of $X$ that we have achieved is equivariant. This follows now from 3.3, together with the equivariant resolution of the basic object $\left(W_{0},\left(J_{0}, 1\right), E_{0}\right)$ provided by the algorithm of resolution of basic objects.

\section{Extension of the theorem of algorithmic desingular- ization}

Our proof of desingularization in 4.8 extends to an ample class of schemes and analytic spaces. This point has been addressed in [15] (see 5.11), and also in section 8 of [11]. Here we will only discuss the extension of our proof to locally embedded schemes.

5.1. We refer to $[16,6.9]$ for properties of the algorithm of resolution of basic objects, proved in [16, Theorem 6.13]. The following are two examples, from which we conclude properties on the desingularization (5.2). Fix a basic object $\left(W_{0},\left(J_{0}, b\right), E_{0}\right)$.

1. Let $W_{0}^{\prime} \longrightarrow W_{0}$ be an étale morphism and, $J_{0}^{\prime} \subset \mathcal{O}_{W_{0}^{\prime}}$ and $E_{0}^{\prime}$ the pullbacks of $J_{0}$ and $E_{0}$; so that $\left(W_{0}^{\prime},\left(J_{0}^{\prime}, b\right), E_{0}^{\prime}\right)$ is a basic object. Then the algorithmic resolution of $\left(W_{0}^{\prime},\left(J_{0}^{\prime}, b\right), E_{0}^{\prime}\right)$ is the pull-back of that of $\left(W_{0},\left(J_{0}, b\right), E_{0}\right)$.

2. Let $W_{0}^{\prime} \longrightarrow W_{0}$ be defined by an arbitrary extension of the base field and consider the pull-back $\left(W_{0}^{\prime},\left(J_{0}^{\prime}, b\right), E_{0}^{\prime}\right)$. Then the algorithmic resolution of $\left(W_{0}^{\prime},\left(J_{0}^{\prime}, b\right), E_{0}^{\prime}\right)$ is also the pull-back of the resolution of $\left(W_{0},\left(J_{0}, b\right), E_{0}\right)$.

5.2. The following properties hold for theorem 1.5 .

1. Let $W_{0}^{\prime} \longrightarrow W_{0}$ be an étale morphism and $X_{0}^{\prime} \subset W_{0}^{\prime}$ the pull-back of $X_{0}$. Then the algorithmic desingularization $\left(X_{r}^{\prime} \subset W_{r}^{\prime}\right) \longrightarrow\left(X_{0}^{\prime} \subset W_{0}^{\prime}\right)$ is the pull-back of that of $\left(X_{r} \subset W_{r}\right) \longrightarrow\left(X_{0} \subset W_{0}\right)$. 
2. If $W_{0}^{\prime} \longrightarrow W_{0}$ is defined by an arbitrary extension of the base field, then the algorithmic desingularization $\left(X_{r}^{\prime} \subset W_{r}^{\prime}\right) \longrightarrow\left(X_{0}^{\prime} \subset W_{0}^{\prime}\right)$ is the pull-back of that of $\left(X_{r} \subset W_{r}\right) \longrightarrow\left(X_{0} \subset W_{0}\right)$.

They both follow from the algorithmic proof in 4.8 and 5.1 .

5.3. Extension to locally embedded schemes. The embedded desingularization in theorem 1.5 defines a non-embedded desingularization, namely a proper birational morphism $X_{r} \longrightarrow X_{0}$, where $X_{r}$ is regular, and the morphism is an isomorphism over $\operatorname{Reg}\left(X_{0}\right)$. Let us show that our procedure of desingularization will also define non-embedded desingularization for schemes which can be locally embedded in smooth schemes. This is always the case for a noetherian separated scheme $X_{0}$, of finite type over a field $\mathbf{k}$ of characteristic zero. It suffices to prove that for two embeddings of $X_{0}$ (local embeddings) we obtain the same non-embedded desingularization. We first address the following lemma, also important for the study of equivariance for non-embedded desingularization:

Lemma 5.4. Let $W_{0}$ and $W_{0}^{\prime}$ be pure dimensional schemes, smooth over $\mathbf{k}$, with $\operatorname{dim} W_{0}=\operatorname{dim} W_{0}^{\prime}$, and let $J_{0} \subset \mathcal{O}_{W_{0}}$ and $J_{0}^{\prime} \subset \mathcal{O}_{W_{0}^{\prime}}$, be two sheaves of ideals. Assume that for two points $\xi_{0} \in W_{0}$ and $\xi_{0}^{\prime} \in W_{0}^{\prime}$ that there is an isomorphism $\Theta: \widehat{\mathcal{O}}_{W_{0}, \xi_{0}} \longrightarrow \widehat{\mathcal{O}}_{W_{0}^{\prime}, \xi_{0}^{\prime}}$ such that $\Theta\left(\widehat{J}_{0}\right)=\widehat{J}_{0}^{\prime}$ (where $\widehat{\mathcal{O}}_{W_{0}, \xi_{0}}$ and $\widehat{\mathcal{O}}_{W_{0}^{\prime}, \xi_{0}^{\prime}}$ denote the completions of $\mathcal{O}_{W_{0}, \xi_{0}}$ and $\mathcal{O}_{W_{0}^{\prime}, \xi_{0}^{\prime}}$ respectively, and $\left.\widehat{J}_{0}=J_{0} \widehat{\mathcal{O}}_{W_{0}, \xi_{0}}, \widehat{J}_{0}^{\prime}=J_{0}^{\prime} \widehat{\mathcal{O}}_{W_{0}^{\prime}, \xi_{0}^{\prime}}\right)$.

Then there is a common étale neighborhood $\tilde{\xi}_{0} \in \widetilde{W}_{0}$ of both $\xi_{0}$ and $\xi_{0}^{\prime}$, and and an ideal $\widetilde{J}_{0} \subset \mathcal{O}_{\widetilde{W}_{0}}$ such that

$$
\widetilde{J}_{0}=J_{0} \mathcal{O}_{\widetilde{W}_{0}}=J_{0}^{\prime} \mathcal{O}_{\widetilde{W}_{0}}
$$

Proof: If $\Theta$ arises from an isomorphism $\Theta: W_{0} \rightarrow W_{0}^{\prime}$ mapping $\xi_{0} \in W_{0}$ to $\xi_{0}^{\prime} \in W_{0}^{\prime}$, and $\Theta\left(J_{0}\right)=J_{0}^{\prime}$, then it is enough to take $\widetilde{W}_{0}=W_{0}$ (note that an isomorphism is an étale map).

We claim that this is the case in general, at least replacing $W_{0}$ and $W_{0}^{\prime}$ by suitable étale neighborhoods in the given points: Since the local rings $\mathcal{O}_{W_{0}, \xi_{0}} /\left(J_{0}\right)_{\xi}$ and $\mathcal{O}_{W_{0}^{\prime}, \xi_{0}^{\prime}} /\left(J_{0}^{\prime}\right)_{\xi^{\prime}}$ are formally isomorphic, then their henselizations are isomorphic (see $[5,2.6])$.

Now, an isomorphism of these henselizations can be lifted to an isomorphism of the henselizations of the regular local rings, say $\Gamma: \overline{\mathcal{O}}_{W_{0}, \xi_{0}} \rightarrow$ $\overline{\mathcal{O}}_{W_{0}^{\prime}, \xi_{0}^{\prime}}$, mapping $J_{0} \overline{\mathcal{O}}_{W_{0}, \xi_{0}}$ to $J_{0}^{\prime} \overline{\mathcal{O}}_{W_{0}^{\prime}, \xi_{0}^{\prime}}$. Since both henselizations are direct limits of étale neighborhoods, $\Gamma$ also defines an isomorphism, as indicated above, at suitable étale neighborhoods. 
Proposition 5.5. Let $X_{0}$ be a noetherian separated scheme of finite type over k. Consider two closed immersions $X_{0} \subset W_{0}$ and $X_{0} \subset W_{0}^{\prime}$, where $W_{0}$ and $W_{0}^{\prime}$ are pure dimensional smooth schemes over $\mathbf{k}$. The embedded desingularizations (1.5):

$$
\begin{aligned}
& \left(X_{0} \subset W_{0}, \emptyset\right) \longleftarrow\left(X_{r} \subset W_{r}, E_{r}\right) \\
& \left(X_{0} \subset W_{0}^{\prime}, \emptyset\right) \longleftarrow\left(X_{r^{\prime}}^{\prime} \subset W_{r^{\prime}}^{\prime}, E_{r^{\prime}}^{\prime}\right)
\end{aligned}
$$

define non-embedded desingularizations $\varphi: X_{r} \longrightarrow X_{0}$ and $\varphi^{\prime}: X_{r^{\prime}}^{\prime} \longrightarrow X_{0}$.

Then $X_{r}=X_{r^{\prime}}^{\prime}$ and $\varphi=\varphi^{\prime}$. Moreover the number of blowing ups also coincide (i.e., $r=r^{\prime}$ ).

Proof: Fix a point $x_{0} \in X_{0}$, which will defines $\xi_{0} \in W_{0}$ and $\xi_{0}^{\prime} \in W_{0}^{\prime}$. Since étale maps are open, it suffices to prove, that for any $x_{0} \in X_{0}$ there is an étale neighborhood where the pull-back of both $X_{0} \longleftarrow X_{r}$ and $X_{0} \longleftarrow X_{r^{\prime}}^{\prime}$ coincide.

Case $\mathbf{A}\left(n=\operatorname{dim}\left(W_{0}\right)=\operatorname{dim}\left(W_{0}^{\prime}\right)\right)$. Consider now the two short exact sequences

$$
\begin{aligned}
0 \longrightarrow J_{0} \longrightarrow \mathcal{O}_{W_{0}, \xi_{0}} \longrightarrow \mathcal{O}_{X_{0}, x_{0}} \longrightarrow 0 \\
0 \longrightarrow J_{0}^{\prime} \longrightarrow \mathcal{O}_{W_{0}^{\prime}, \xi_{0}^{\prime}} \longrightarrow \mathcal{O}_{X_{0}, x_{0}} \longrightarrow 0
\end{aligned}
$$

Identify now $S$ with the completion of both, the local regular rings $\mathcal{O}_{W_{0}, \xi_{0}}$ and $\mathcal{O}_{W_{0}^{\prime}, \xi_{0}^{\prime}}$. We shall define an isomorphism $\Theta \in \operatorname{Aut}(S)$ so that

$$
\Theta\left(J_{0} S\right)=J_{0}^{\prime} S
$$

The assertion, within case A, would then follow from 5.4. Let $z_{1}, \ldots, z_{n}$ be a regular system of parameters of $\mathcal{O}_{W_{0}, \xi_{0}}$ mapping to $y_{1}, \ldots, y_{n}$ in $\mathcal{O}_{X_{0}, x_{0}}$, and let $z_{1}^{\prime}, \ldots, z_{n}^{\prime}$ be a regular system of parameters in $\mathcal{O}_{W_{0}^{\prime}, \xi_{0}^{\prime}}$ mapping to $y_{1}^{\prime}, \ldots, y_{n}^{\prime}$ in $\mathcal{O}_{X_{0}, x_{0}}$. If $d$ denotes the embedded dimension of $X_{0}$ at $\xi_{0}$, we may assume that both regular systems of coordinates are chosen so that $z_{d+1}, \ldots, z_{n}$ and $z_{d+1}^{\prime}, \ldots, z_{n}^{\prime}$ map to zero at $\mathcal{O}_{X_{0}, x_{0}}$. So that $y_{1}, \ldots, y_{d}$ and $y_{1}^{\prime}, \ldots, y_{d}^{\prime}$ are both generators of the maximal ideal $\mathfrak{m}_{X_{0}, x_{0}}$ of $\mathcal{O}_{X_{0}, x_{0}}$.

There exists $g_{i, j}^{\prime} \in \mathcal{O}_{X_{0}, x_{0}}$ such that

$$
y_{i}=g_{i, 1}^{\prime} y_{1}^{\prime}+\cdots+g_{i, d}^{\prime} y_{d}^{\prime} \quad i=1, \ldots, d
$$

Since the classes of $y_{1}, \ldots, y_{d}$ and $y_{1}^{\prime}, \ldots, y_{d}^{\prime}$ are both basis of $\mathfrak{m}_{X_{0}, x_{0}} / \mathfrak{m}_{X_{0}, x_{0}}^{2}$, the determinant of the matrix $\left(g_{i, j}^{\prime}\right)_{i, j=1}^{d}$ is a unit in $\mathcal{O}_{X_{0}, x_{0}}$.

Choose elements $f_{i, j}^{\prime} \in \mathcal{O}_{W_{0}^{\prime}, \xi_{0}^{\prime}}$ which map to $g_{i, j}^{\prime}$. Note that the determinant of the matrix $\left(f_{i, j}^{\prime}\right)_{i, j=1}^{d}$ is also a unit of $\mathcal{O}_{W_{0}^{\prime}, \xi_{0}^{\prime}}$. 
Finally define a morphism $\Theta: S \longrightarrow S$ by setting $\Theta\left(z_{i}\right)=f_{i, 1}^{\prime} z_{1}^{\prime}+$ $\cdots+f_{i, d}^{\prime} z_{d}^{\prime}$ for $i=1, \ldots, d$, and $\Theta\left(z_{i}\right)=z_{i}^{\prime}$ for $i=d+1, \ldots, n$. Now it can be checked that $\Theta$ is an isomorphism, it induces the identy map at the completion of $\mathcal{O}_{X_{0}, x_{0}}$, and hence it fulfills 5.5.1.

Now the result follows from 5.4 and 5.2.

Case B $\left(\operatorname{dim} W_{0}<\operatorname{dim} W_{0}^{\prime}\right)$. We proceed by induction on $m=\operatorname{dim} W_{0}^{\prime}-$ $\operatorname{dim} W_{0}$.

Note that the embedding dimension of $X_{0}$ at $\xi_{0}^{\prime}$ is smaller than $\operatorname{dim} W_{0}^{\prime}$. So locally at $\xi_{0}^{\prime}$ there is a smooth hypersurface $W_{0}^{\prime \prime} \subset W_{0}^{\prime}$ and $X_{0} \subset W_{0}^{\prime \prime}$. The embedded desingularization of $X_{0} \subset W_{0}^{\prime}$ is obtained from the resolution of the basic object $\left(W_{0}^{\prime},\left(\mathcal{I}_{W_{0}^{\prime}}\left(X_{0}\right), 1\right), E_{0}^{\prime}\right)$ (see 4.8$)$, where $E_{0}^{\prime}=\emptyset$ and $\mathcal{I}_{W_{0}^{\prime}}\left(X_{0}\right) \subset \mathcal{O}_{W_{0}^{\prime}}$ is the ideal of $X_{0}$ in $W_{0}^{\prime}$. Note that $\mathcal{I}_{W_{0}^{\prime}}\left(W_{0}^{\prime \prime}\right) \subset \mathcal{I}_{W_{0}^{\prime}}\left(X_{0}\right)$. So that the maximum order of the ideal $\mathcal{I}_{W_{0}^{\prime}}\left(X_{0}\right)$ is one. In this case the algorithm of resolution for basic objects defines a basic object in dimension $\operatorname{dim} W_{0}^{\prime}-1$, say:

$$
\left(W_{0}^{\prime \prime},\left(\operatorname{Coeff}_{W_{0}^{\prime \prime}}\left(\mathcal{I}_{W_{0}^{\prime}}\left(X_{0}\right)\right), 1\right), E_{0}^{\prime \prime}\right)
$$

with $E_{0}^{\prime \prime}=\emptyset$ (see [17, Lemma 6.12]). Where $\operatorname{Coeff}_{W_{0}^{\prime \prime}}\left(\mathcal{I}_{W_{0}^{\prime}}\left(X_{0}\right)\right)$ is the coefficient ideal ([17, Definition 9.3]). And it follows from definition of the coefficient ideal that in this case:

$$
\operatorname{Coeff}_{W_{0}^{\prime \prime}}\left(\mathcal{I}_{W_{0}^{\prime}}\left(X_{0}\right)\right)=\mathcal{I}_{W_{0}^{\prime \prime}}\left(X_{0}\right) .
$$

So that the resolution of the basic objects

$$
\left(W_{0}^{\prime},\left(\mathcal{I}_{W_{0}^{\prime}}\left(X_{0}\right), 1\right), E_{0}^{\prime}\right) \quad \text { and } \quad\left(W_{0}^{\prime \prime},\left(\mathcal{I}_{W_{0}^{\prime \prime}}\left(X_{0}\right), 1\right), E_{0}^{\prime \prime}\right)
$$

are the same ([17, Lemma 6.12]). But by induction on $m$ the resolution of the basic objects

$$
\left(W_{0}^{\prime \prime},\left(\mathcal{I}_{W_{0}^{\prime \prime}}\left(X_{0}\right), 1\right), E_{0}^{\prime \prime}\right) \quad \text { and } \quad\left(W_{0},\left(\mathcal{I}_{W_{0}}\left(X_{0}\right), 1\right), E_{0}\right)
$$

are also equal, and the result follows in Case B.

\section{References}

[1] Abramovich, D. And De Jong, A. J.: Smoothness, semistability and toroidal geometry. J. Algebraic Geom. 6 (1997), no.4, 789-801.

[2] Abramovich, D. And Wang, J.: Equivariant resolution of singularities in characteristic 0. Math. Res. Lett. 4 (1997), no. 2-3, 427-433.

[3] Aroca, J. M., Hironaka, H. and Vicente, J. L.: The theory of maximal contact, Memorias Matemáticas Instituto Jorge Juan 29, Consejo Superior de Investigaciones Científicas, 1975. 
[4] Aroca, J. M., Hironaka, H. and Vicente, J. L.: Desingularization theorems, Memorias de Matemáticas del Instituto Jorge Juan 30, Consejo Superior de Investigaciones Científicas, 1977.

[5] Artin, M.: Algebraic approximation of structures over complete local rings. Inst. Hautes Études Sci. Publ. Math. 36 (1969), 23-58.

[6] Berthelot, P.: Altérations des variétés algebriques (d'après A. J. de Jong). Asterisque 241 (1997), Exp. no. 815, 5, 273-311.

[7] Bierstone, E. And Milman, P.: Canonical desingularization in characteristic zero by blowing-up the maximal strata of a local invariant. Invent. Math. 128 (1997), no. 2, 207-302.

[8] Bodnár, G. And Schicho, J.: Automated resolution of singularities for hypersurfaces. J. Symbolic Comput. 30 (2000), no. 4, 401-428.

[9] Bodnár, G. ANd Schicho, J.: A computer program for the resolution of singularities. In Resolution of singularities (Obergurgl, 1997), 231-238. Progr. Math. 181, Birkhäuser, Basel, 2000.

[10] Bogomolov, F. And Pantev, T.: Weak hironaka theorem. Math. Res. Lett. 3 (1996), 299-307.

[11] Bravo, A., Encinas, S. and Villamayor, O.: A Simplified proof of desingularization and applications. To appear in Rev. Mat. Iberoamericana. A copy is available at http://arXiv.org/abs/math.AG/0206244.

[12] Bravo, A. and Villamayor, O.: Strengthening a Theorem of Embedded Desingularization. Math. Res. Lett. 8 (2001), 1-11.

[13] Bravo, A. And Villamayor, O.: A Strengthening of resolution of singularities in characteristic zero. Proc. London Math. Soc. 86 (2003), no. 2, 327-357.

[14] Encinas, S. And Hauser, H.: Strong resolution of singularities. Coment. Math. Helv. 77 (2002), no. 4, 821-845.

[15] Encinas, S., Nobile, A. and Villamayor, O.: On algorithmic equiresolution and stratification of Hilbert Schemes. Proc. London Math. Soc. 86 (2003), no. 3, 607-648.

[16] Encinas, S. and Villamayor, O.: Good points and constructive resolution of singularities. Acta Math. 181 (1998), no. 1, 109-158.

[17] Encinas, S. And Villamayor, O.: A course on constructive desingularization and equivariance. In Resolution of singularities (Obergurgl, 1997), 147-227. Progr. Math. 181, Birkhäuser, Basel, 2000.

[18] Giraud, J.: Sur la théorie du contact maximal. Math. Z. 137 (1974), $285-310$.

[19] Giraud, J.: Contact maximal en caractéristique positive. Ann. Sci. École Norm. Sup. (4) 8 (1975), 201-234.

[20] Hironaka, H.: Resolution of singularities of an algebraic variety over a field of characteristic zero: I, II. Ann. of Math. 79 (1964), no. 1-2, 109-326. 
[21] Hironaka, H.: Idealistic exponents of singularity. In Algebraic geometry (J. J. Sylvester Sympos., Johns Hopkins Univ., Baltimore, Md., 1976), 52125. Johns Hopkins Univ. Press, Baltimore, Md., 1977.

[22] Lejeune-Jalabert, M. And Teissier, B.: Quelques calculs utiles pour la résolution des singularités. Exposés faits au Centre de Mathématiques de l'École Polytechnique, no. M72.1171, Centre de Mathématiques, École Polytechnique, Paris, 1971.

[23] Lipman, J.: Rational singularities, with applications to algebraic surfaces and unique factorization. Inst. Hautes Études Sci. Publ. Math. 36 (1969), $195-279$

[24] Matsuki, K.: Notes on the inductive algorithm of resolution of singularities by S. Encinas and O. Villamayor. Preprint, available at http://arXiv.org/abs/math.AG/0103120.

[25] Matsumura, H.: Commutative algebra. Mathematics Lecture Note Series 56, Benjamin/Cummings Publishing Co., Inc., Reading, Mass., 1980.

[26] OdA, T.: Infinitely very near singular points. In Complex analytic singularities, 363-404. Adv. Studies in Pure Math. 8, North-Holland, 1987.

[27] Villamayor, O.: Constructiveness of Hironaka's resolution. Ann. Sci. École Norm. Sup. (4) 22 (1989), no. 1, 1-32.

[28] Villamayor, O.: Patching local uniformizations. Ann. Sci. École Norm. Sup. (4) 25 (1992), no. 6, 629-677.

[29] Villamayor, O.: Introduction to the algorithm of resolution. In Algebraic geometry and singularities (La Rabida, 1991), 123-154. Progr. Math. 134, Birkhäuser, 1996.

Recibido: 18 de marzo de 2002

Revisado: 7 de septiembre de 2002

Santiago Encinas

Departamento de Matemática Aplicada Fundamental

ETS Arquitectura

Universidad de Valladolid

Avda. Salamanca, s/n, 47014 Valladolid, Spain

sencinas@maf .uva.es

Orlando Villamayor

Departamento de Matemáticas

Universidad Autónoma de Madrid

Campus de Cantoblanco, s/n, 28049 Madrid, Spain

villamayor@uam.es

Both authors were partially supported by DGES, BFM2000-0026. 